\title{
HS 0139+0559, HS 0229+8016, HS 0506+7725, and HS 0642+5049: four new long-period cataclysmic variables ${ }^{\star} \star \star$
}

\author{
A. Aungwerojwit ${ }^{1}$, B. T. Gänsicke ${ }^{1}$, P. Rodríguez-Gil ${ }^{1,2}$, H.-J. Hagen ${ }^{3}$, E. T. Harlaftis ${ }^{4}$, C. Papadimitriou ${ }^{5,6}$, \\ H. Lehto ${ }^{7,8}$, S. Araujo-Betancor ${ }^{9}$, U. Heber ${ }^{10}$, R. E. Fried ${ }^{11}$, D. Engels ${ }^{3}$, and S. Katajainen ${ }^{8}$
}

1 Department of Physics, University of Warwick, Coventry CV4 7AL, UK

e-mail: A. Aungwerojwit@warwick.ac.uk

2 Instituto de Astrofísica de Canarias, 38200 La Laguna, Tenerife, Spain

3 Hamburger Sternwarte, Universität Hamburg, Gojenbergsweg 112, 21029 Hamburg, Germany

${ }^{4}$ Institute of Space Applications and Remote Sensing, National Observatory of Athens, PO Box 20048, Athens 11810, Greece

5 Institute of Astronomy and Astrophysics, National Observatory of Athens, PO Box 20048, Athens 11810, Greece

${ }^{6}$ Department of Astrophysics, Astronomy and Mechanics, University of Athens, 15784 Zografos, Athens, Greece

7 Department of Physics, 20014 University of Turku, Finland

8 Tuorla Observatory, University of Turku, 21500 Piikkiö, Finland

9 Space Telescope Science Institute, 3700 San Martin Drive, Baltimore, MD21218, USA

10 Astronomisches Institut der Universität Erlangen, Germany

11 Braeside Observatory, PO Box906, Flagstaff, AZ 86002, USA

Received 23 December 2004 / Accepted 3 July 2005

\section{ABSTRACT}

We present time-resolved optical spectroscopy and photometry of four relatively bright $(V \sim 14.0-15.5)$ long-period cataclysmic variables (CVs) discovered in the Hamburg Quasar Survey: HS 0139+0559, HS 0229+8016, HS 0506+7725, and HS 0642+5049. Their respective orbital periods, $243.69 \pm 0.49 \mathrm{~min}, 232.550 \pm 0.049 \mathrm{~min}, 212.7 \pm 0.2 \mathrm{~min}$, and $225.90 \pm 0.23 \mathrm{~min}$ are determined from radial velocity and photometric variability studies. HS 0506+7725 is characterised by strong Balmer and He emission lines, short-period ( 10-20 min) flickering, and weak X-ray emission in the ROSAT All Sky Survey. The detection of a deep low state $(B \simeq 18.5)$ identifies HS 0506+7725 as a member of the VY Scl stars. HS 0139+0559, HS 0229+8016, and HS 0642+5049 display thick-disc like spectra and no or only weak flickering activity. HS 0139+0559 and HS 0229+8016 exhibit clean quasi-sinusoidal radial velocity variations of their emission lines but no or very little orbital photometric variability. In contrast, we detect no radial velocity variation in HS $0642+5049$ but a noticeable orbital brightness variation. We identify all three systems either as UX UMa-type novalike variables or as Z Cam-type dwarf novae. Our identification of these four new systems underlines that the currently known sample of CVs is rather incomplete even for bright objects. The four new systems add to the clustering of orbital periods in the 3-4 h range found in the sample of HQS selected CVs, and we discuss the large incidence of magnetic CVs and VY Scl/SW Sex stars found in this period range among the known population of CVs.

Key words. stars: binaries: close - stars: individual: HS 0139+0559 - stars: individual: HS 0229+8016 - stars: dwarf novae stars: individual: HS 0506+7725 - stars: individual: HS 0642+5049 - stars: novae, cataclysmic variables

\section{Introduction}

Standard models for the population of cataclysmic variables (CVs) predict that the vast majority of all CVs should have

* Based on observations obtained at the German-Spanish Astronomical Center, Calar Alto, operated by the Max-PlanckInstitut für Astronomie, Heidelberg, jointly with the Spanish National Commission for Astronomy, on observations made at the $1.2 \mathrm{~m}$ telescope, located at Kryoneri Korinthias, and owned by the National Observatory of Athens, Greece, and on observations made with the OGS telescope, operated on the island of Tenerife by the European Space Agency, in the Spanish Observatorio del Teide of the IAC.

$\star \star$ Table 1 is only available in electronic form at http://www. edpsciences.org short orbital periods, $P_{\text {orb }}<2$ h (e.g. Kolb 1993; Howell et al. 1997), and a space density of $2 \times 10^{-5}-2 \times 10^{-4} \mathrm{pc}^{-3}$ (Ritter \& Burkert 1986; de Kool 1992; Politano 1996). These predictions contrast with the properties of the observed population of galactic CVs, with an estimated space density of $\sim 6 \times 10^{-6} \mathrm{pc}^{-3}$ (Ringwald 1996; Araujo-Betancor et al. 2005b) and an apparent lack of short-period systems. Possible reasons for these discrepancies are uncertainties in the theory of $\mathrm{CV}$ evolution (e.g. King 1988; Schenker \& King 2002; Andronov et al. 2003; Barker \& Kolb 2003; Taam et al. 2003), but also observational selection effects in the known CV population (e.g Downes 1986; Ringwald 1996; Gänsicke 2005).

About $75 \%$ of all known CVs have been discovered either because of their variability or because of their X-ray emission, 
with a strong dominance of dwarf novae and classical novae in the first group and magnetic CVs in the second group (Gänsicke 2005). It is therefore clear that CVs characterised by infrequent outbursts and/or low-amplitude variability, as well as lacking strong X-ray emission, will be underrepresented in the currently known CV population. Hypothetically, such objects could make up for the large number of predicted shortperiod CVs with low mass transfer rates.

We are currently carrying out a spectroscopic survey of CV candidates with the aim to produce a homogeneously selected sample of CVs that overcomes the observational biases mentioned above. In this paper, we describe the status of our survey, the identification of four new CVs with periods close to $4 \mathrm{~h}$, and discuss the nature of CVs in the 3-4 h period range.

\section{CVs in the Hamburg Quasar Survey}

The primary purpose of our project to identify new CVs in the Hamburg Quasar Survey (HQS) is to establish a large homogeneously selected sample of systems that overcomes previous observational biases and that can subsequently be used to test our understanding of CV evolution (Gänsicke et al. 2002b). The HQS, an objective-prism survey, was carried out with the $0.8 \mathrm{~m}$ Schmidt telescope at Calar Alto Observatory to search for bright quasars in the northern sky at high galactic lattitudes, $\delta>0^{\circ}$ and $|b|>20^{\circ}$, covering $\approx 13600 \mathrm{deg}^{2}$, with a dynamic range of $13 \lesssim B \lesssim 18.5$ (Hagen et al. 1995). The photographic plates cover a spectral range of $\sim 3400-5400 \AA$ with a resolution of $\sim 45 \AA$ at $\mathrm{H} \beta$.

Our CV candidate selection made use of a property that has never been systematically exploited before: the spectroscopic hallmark of CVs, i.e. the presence of noticeable emission lines in most CVs. The CV candidate selection was carried out by visually inspecting $48708 \mathrm{HQS}$ prism spectra for the presence of Balmer emission lines. In order to test the efficiency of this method, we applied the same procedure to the subset of 84 previously known CVs (Downes et al. 2001, as of July 2001) that are contained in the HQS spectral data base. We positively recovered $\simeq 90 \%$ of the known short-period $\left(P_{\text {orb }}<2\right.$ h) CVs, including prominent dwarf novae such as SW UMa or T Leo (the latter one has a rather long outburst cycle of $\sim 420 \mathrm{~d}$ ), as well as magnetic CVs, such as AN UMa or STLMi. The fraction of recovered systems drops to $\simeq 40 \%$ for long-period systems, with the largest fraction of missed identifications being novalike variables with weak or no Balmer emission lines. In total, 62\% of the previously known CVs with an HQS prism spectrum were positively identified by our selection method. We concluded from this test that the HQS should be very efficient in finding CVs below the period gap, as long as they have similar spectroscopic properties to the previously known systems, i.e. Balmer emission lines with equivalent widths $>10 \AA$ in $\mathrm{H} \beta$. The decrease in detection efficiency for long-period systems has been compensated to some extent by follow-up programs investigating hot stars in the HQS, delivering a number of new CVs with weak emission lines (Heber et al. 1991).

In total, 53 new CVs were identified within this project, and substantial observational effort was invested in determining the properties of these systems. To date, $42 \mathrm{HQS} \mathrm{CVs} \mathrm{have}$ had their orbital period measured. Despite its good sensitivity for short-period CVs, only a small number of new short-period CVs have been found, and those that have been found fully confirm the expected properties: low-amplitude variability and/or long outburst recurrence times, e.g. KV Dra (HS 1449+6415, Nogami et al. 2000), HS 2331+3905 (Araujo-Betancor et al. 2005a), DW Cnc (HS 0756+1624, Rodríguez-Gil et al. 2004a), or HS 2219+1824 (Rodríguez-Gil et al. 2005a). The majority of the newly identified systems have orbital periods above the 2-3 $\mathrm{h}$ period gap, including rarely outbursting dwarf novae such as GY Cnc (HS 0907+1902, Gänsicke et al. 2000) or RX J0944.5+0357 (HS0941+0411, Mennickent et al. 2002); magnetic CVs with relatively weak X-ray emission, such as 1RXS J062518.2+733433 (HS0618+7336, AraujoBetancor et al. 2003), RX J1554.2+2721 (HS 1552+2730, Thorstensen \& Fenton 2002; Gänsicke et al. 2004b), and HS 0943+1404 (Rodríguez-Gil et al. 2005b); and half a dozen new SW Sextantis stars (Gänsicke et al. 2002c; Szkody et al. 2001; Rodríguez-Gil et al. 2004b; Rodríguez-Gil 2005).

While a full discussion of the implications that our search for CVs in the HQS has for our understanding of the galactic $\mathrm{CV}$ population has to await characterisation of the full HQS CV sample, an important preliminary statement that will not substantially change is: there is no large population of nearby short-period CVs that resemble the known template systems. Phrased differently, if the large population of short period CVs predicted by theory exists, the majority of these systems must look different from the known short-period systems, i.e. have weak emission lines and/or substantially redder continua. Interestingly, the Sloan Digital Sky Survey (SDSS) is discovering a number of $\mathrm{CVs}$ with a steep Balmer decrement, in which the white dwarf dominates the optical emission, a clear sign of low mass transfer rates (Szkody et al. 2005, and references therein). However, most of these systems are very faint, $g \simeq 19-20$, implying that they are distant $(d>100 \mathrm{pc})$ and not intrinsically numerous anywhere near the numbers predicted by theory.

An unexpected finding of our search for CVs in the HQS has been the identification of a large number of systems with orbital periods in the range 3-4 h. Here, we report the discovery of four additional CVs in that period range, HS 0139+0559, HS 0229+8016, HS 0506+7725, and HS 0642+5049. Despite being close in orbital period, these systems differ dramatically in their observed characteristic, and we discuss in detail the properties of the known CVs in the 3-4 h period range.

\section{Observations and data reduction}

\subsection{Spectroscopy}

An identification spectrum of HS 0139+0559 (see finding chart in Fig. 1) was obtained in October 1989 at the Calar Alto $3.5 \mathrm{~m}$ telescope using the Boller\&Chivens spectrograph equipped with a $120 \AA / \mathrm{mm}$ grating as part of a program to find blue stars (Heber et al. 1991). This spectrum (Fig. 2) is characterised by a blue continuum with strong absorption lines of the Balmer series, as well as of He I $\lambda 4471$. The absorption profiles have a rectangular shape with a full width at zero intensity (FWZI) 

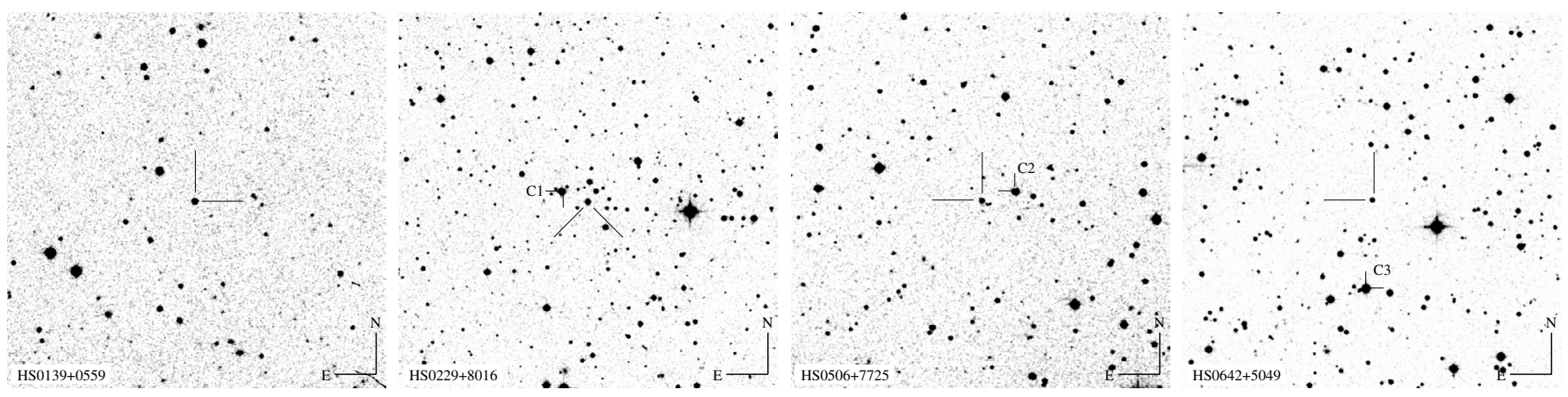

Fig. 1. $10^{\prime} \times 10^{\prime}$ finding charts for HS 0139+0559, HS 0229+8016, HS 0506+7725, and HS 0642+5049 obtained from the Digitized Sky Survey. The J2000 coordinates of the CVs are $\left(\alpha=01^{\mathrm{h}} 41^{\mathrm{m}} 40.0^{\mathrm{s}}, \delta=+06^{\circ} 14^{\prime} 36.8^{\prime \prime}\right),\left(\alpha=02^{\mathrm{h}} 35^{\mathrm{m}} 58.3^{\mathrm{s}}, \delta=+80^{\circ} 29^{\prime} 44.0^{\prime \prime}\right),\left(\alpha=05^{\mathrm{h}} 13^{\mathrm{m}} 36.4^{\mathrm{s}}\right.$, $\left.\delta=+77^{\circ} 28^{\prime} 42.1^{\prime \prime}\right)$, and $\left(\alpha=06^{\mathrm{h}} 46^{\mathrm{m}} 19.60^{\mathrm{s}}, \delta=+50^{\circ} 45^{\prime} 48.0^{\prime \prime}\right)$, respectively.

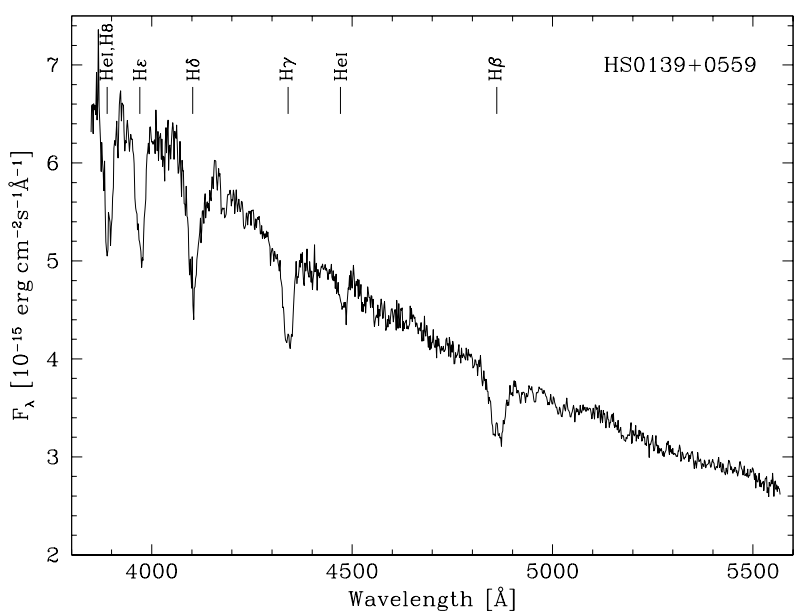

Fig. 2. Identification spectrum of HS $0139+0559$ obtained at the Calar Alto $3.5 \mathrm{~m}$ telescope on January 22, 1989.

of $\sim 3500 \mathrm{~km} \mathrm{~s}^{-1}$. The cores of $\mathrm{H} \beta$ and $\mathrm{H} \gamma$ broad absorptions show weak emission lines. No He II $\lambda 4686$ is observed. Overall, the spectrum resembles that of a high-mass transfer rate accretion disc seen at a moderately low inclination, e.g. a dwarf nova in outburst or a novalike variable.

HS 0229+8016 (Fig. 1) was first spectroscopically observed at the Calar Alto $2.2 \mathrm{~m}$ telescope on August 8, 1992, using the Boller \& Chivens spectrograph. The spectrum (Fig. 3, top panel) shows a blue continuum with the Balmer jump in emission, superimposed by moderately strong Balmer emission lines. The higher Balmer line profiles show evidence of a P-Cygni like structure with blue absorption wings increasing in strength for the higher members of the series. He I $\lambda 4471$ is observed in absorption, and an emission line near $4630 \AA$ is detected, which we tentatively identify as the N/C Bowen blend emission. Rather unusual is, however, the fact that He II $\lambda 4686$ is not detected in emission along with the Bowen blend. HS 0229+8016 was observed again at the Calar Alto $2.2 \mathrm{~m}$ telescope on October 5, 1998, using the Calar Alto Faint Object Spectrograph (CAFOS), on this occasion looking nearly identical to HS 0139+8016 (Fig. 3, bottom panel). The spectral characteristics and the variability clearly identify HS $0229+8016$ as a CV.

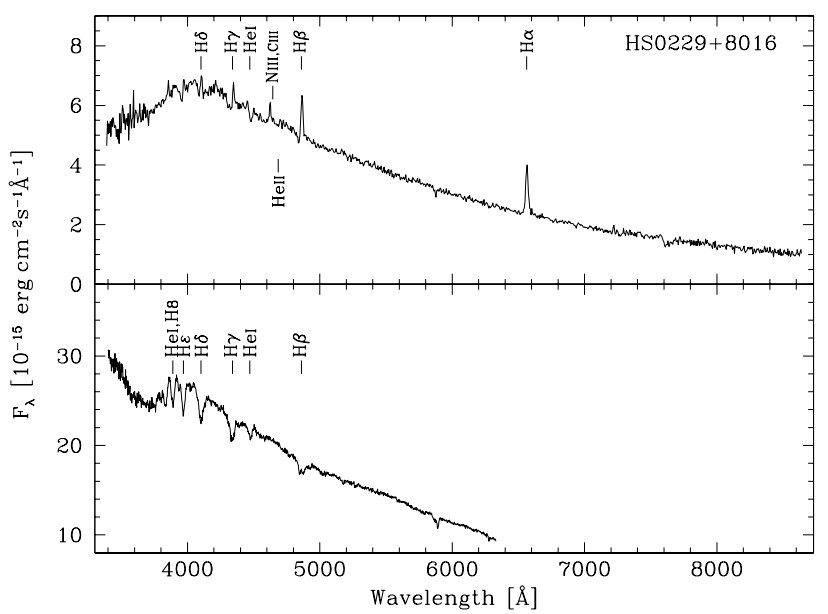

Fig. 3. Identification spectra of HS $0229+8016$ obtained at the Calar Alto $2.2 \mathrm{~m}$ telescope on August 8, 1992 (top panel) and February 2, 1998 (bottom panel).

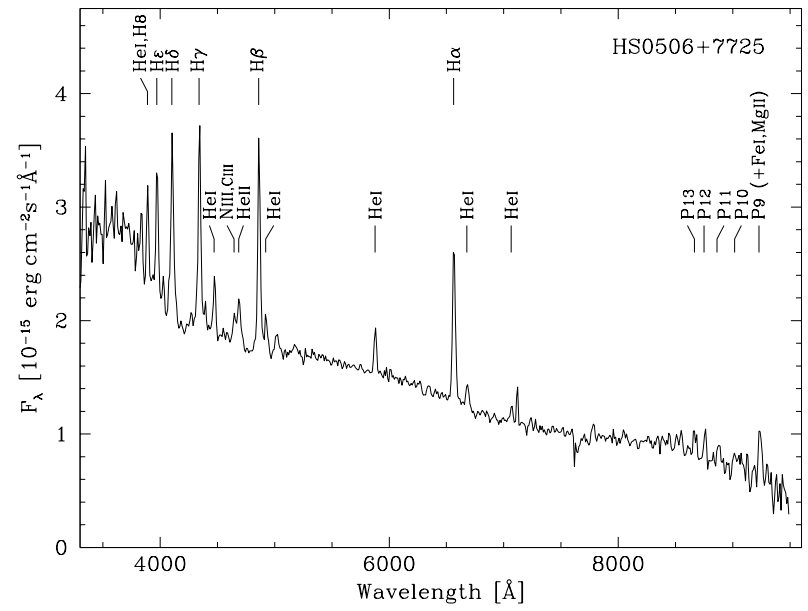

Fig. 4. Identification spectrum of HS $0506+7725$ obtained at the Calar Alto $2.2 \mathrm{~m}$ telescope on February 2, 1998.

An identification spectrum of HS $0506+7725$ (Fig. 1) was obtained on February 2, 1998, on the Calar Alto $2.2 \mathrm{~m}$ telescope using CAFOS with the B-400 grism. The spectrum (Fig. 4) displays a blue continuum with the Balmer and Paschen jumps in emission, plus emission lines of hydrogen and He I. 


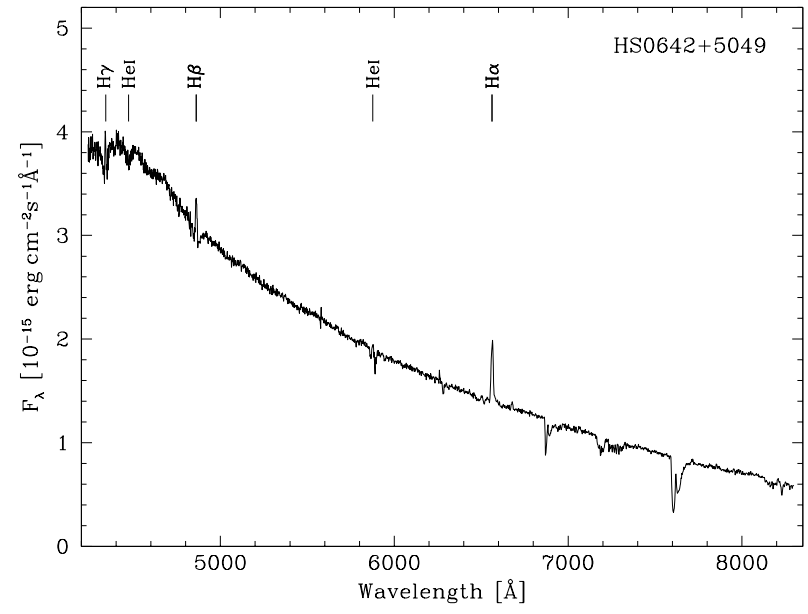

Fig. 5. Average of the 17 CAFOS G-100 spectra of HS 0642+5049 obtained at the Calar Alto $2.2 \mathrm{~m}$ telescope on October 24, 2004.

The $\lambda \lambda 4630-4650$ N/C Bowen blend and the He II $\lambda 4686$ emission lines are also detected. The strength of these highionisation lines is typical of novalike variables, magnetic $\mathrm{CVs}$, or nova remnants.

HS 0642+5049 (Fig. 1) was spectroscopically identified as a CV on March 7, 1999, with CAFOS at the Calar Alto $2.2 \mathrm{~m}$ telescope. The spectrum of HS $0642+5049$ (Fig. 5) contains a blue continuum with moderately strong $\mathrm{H} \alpha$ emission. The $\mathrm{H} \beta$ and $\mathrm{H} \gamma$ emission is embedded in broad absorption troughs, and weak He I $\lambda 4471$ absorption is also detected. No He II $\lambda 4686$ and $\lambda \lambda 4630-4650 \mathrm{~N} / \mathrm{C}$ Bowen blend are detected.

In order to determine the orbital periods of HS $0139+0559$, HS 0229+8016, HS 0506+7725, and HS 0642+5049, we obtained time-resolved spectroscopy at Calar Alto Observatory and Roche de los Muchachos Observatory throughout the period October 2002 to October 2004 (Table 1). At the Calar Alto $2.2 \mathrm{~m}$ telescope, we used the CAFOS spectrograph equipped with the G-100 grating and a $2 \mathrm{k} \times 2 \mathrm{k}$ pixel SITe CCD. This setup, in conjunction with a $1.2^{\prime \prime}$ slit, provided a spectral resolution of $\sim 4.1 \AA$ (full width at half maximum, FWHM) covering the range 4240-8300 $\AA$. We used the double-armed TWIN spectrograph at the Calar Alto $3.5 \mathrm{~m}$ telescope equipped with the T05 grating in the blue and the T06 grating in the red, providing a spectral resolution of $\sim 1.2 \AA(F W H M)$ in the ranges $\lambda \lambda 3810-4940$ and $\lambda \lambda 6440-7510$. At the $2.5 \mathrm{~m}$ Isaac Newton Telescope (INT), we used the Intermediate Dispersion Spectrograph (IDS) equipped with the R632V grating and the $2 \mathrm{k} \times 4 \mathrm{k}$ pixel EEV10a detector. Using a slit width of $1.5^{\prime \prime}$ this setup provided a useful wavelength of 4400-6800 $\AA$ and a spectral resolution of $\sim 2.3 \AA$. Arc calibration spectra were interleaved with the target observations every $\sim 40 \mathrm{~min}$.

In total, we obtained 55 spectra of HS $0139+0559,74$ spectra of HS $0229+8016,86$ spectra of HS 0506+7725, and 87 spectra of HS 0642+5049 (Table 1). Reduction of the follow-up spectroscopy consisting of bias and flat-field corrections and optimal extraction (Horne 1986) was carried out in IRAF. ${ }^{1}$ The wavelength calibration of the extracted spectra was

\footnotetext{
1 IRAF is distributed by the National Optical Astronomy Observatories.
}

performed in MOLLY. The dispersion relation was obtained by fitting a low-order polynomial to the arc lines, with the rms less than one tenth of the dispersion in all cases. The flexure of the telescope was accounted for by interpolating between the two arc exposures bracketing the target spectra.

The time-resolved spectra of HS0139+0559 and HS 0229+8016 are similar to the identification spectra shown in Figs. 2 and 3 (bottom panel), with $\mathrm{H} \beta$ and $\mathrm{H} \gamma$ in absorption for most of the time and occasionally showing signs of emission cores. However, the G-100 spectra cover $\mathrm{H} \alpha$ as well, which is observed in emission throughout, with low equivalent widths in the range 3-6 $\AA$. The time-resolved spectra of HS $0506+7725$ are very similar to the identification spectrum shown in Fig. 4, with the difference of having no $\mathrm{He}$ II $\lambda 4686$ emission. The $\mathrm{H} \alpha$ emission line has an average equivalent width of $\sim 15 \AA$. The time-resolved spectra of HS $0642+5049$ show $\mathrm{H} \alpha$ in emission with an average equivalent width of $\sim 5 \AA$, and weak $\mathrm{H} \beta$ and $\mathrm{H} \gamma$ emission embedded in broad absorption lines (Fig. 5).

\subsection{Photometry}

The spectroscopy of the four new cataclysmic variables was supplemented by differential CCD photometry obtained at six different telescopes (Table 1). Brief descriptions of the instrumentation used and the data reduction techniques are given below.

Braeside Observatory. Differential $B$ and $R$ photometry of HS 0139+0559 was obtained in December 1999/January 2000 at Braeside Observatory using the $0.41 \mathrm{~m}$ reflector together with an SITe $512 \times 512$ pixel CCD camera. The raw images were bias-subtracted, dark current-subtracted, and flat-fielded in a standard manner.

Kryoneri Observatory. We obtained differential $R$-band photometry of HS $0229+8016$ (September 19 and 20, 2002) and HS 0506+7725 (October 8, 2002) at Kryoneri Observatory using the $1.2 \mathrm{~m}$ telescope equipped with a Photometrics SI-502 $516 \times 516$ pixel CCD camera. The data were reduced using the pipeline described in Gänsicke et al. (2004a), which preprocesses the raw images in MIDAS and extracts aperture photometry using the Sextractor (Bertin \& Arnouts 1996). The instrumental magnitudes of HS $0229+8016$ were converted into apparent magnitudes using the comparison star labelled "C1" in Fig. 1 (USNO-A2.0 1650-00512682, $R=12.6$ ). We found a mean magnitude of $R \simeq 14.3$ during both nights. The apparent magnitudes of HS $0506+7725$ were computed using the comparison star "C2" (USNO-A2.0 1650-00942250, $R=13.1$ ), and an average magnitude of $R \simeq 15.2$ was found.

Tuorla Observatory. We used the $0.7 \mathrm{~m}$ Schmidt-Vaisala telescope at Tuorla Observatory, equipped with an SBIG ST-8 CCD camera to obtain filterless photometry of HS $0229+8016$ (January 10, 2003), HS 0506+7725 (January 4, 6, and 16, 2003), and HS 0642+5049 (December 30, 2003 and January 1, 
2004). The reduction of the observations was carried out in the same way as described for the Kryoneri data. Using the same comparison stars as above, we found the mean magnitudes of HS $0229+8016$ and HS $0506+7725$ to be $\simeq 14.3$ and $\simeq 15.8$, respectively. The apparent magnitudes of HS $0642+5049$ were extracted using the comparison star "C3" in Fig. 1 (USNOA2.0 1350-06806656, $R=12.4$ ), and gave a mean magnitude of $\simeq 15.5$.

Observatorio del Teide. At the Observatorio del Teide on Tenerife, we used the $1 \mathrm{~m}$ Optical Ground Station (OGS) and the $0.82 \mathrm{~m}$ IAC80 telescopes to obtain CCD photometry of HS $0506+7725$ and HS $0642+5049$. The telescopes were equipped with Thomson $1 \mathrm{k} \times 1 \mathrm{k}$ pixel CCD cameras. On November 15, 2003, the OGS was used to obtain differential photometry of HS $0506+7225$ in white light. The data were taken using $2 \times 2$ binning and windowing to improve the time resolution. The images were bias and flat-field corrected and aligned within IRAF. Instrumental magnitudes of the CV and the comparison star "C2" were then extracted using the point spread function (PSF) photometry tasks package within IRAF. We found the mean magnitude of HS 0506+7725 during that night to be $\simeq 14.6$. On December 1, 6, 7, and 8, 2004, we used the IAC80 telescope to obtain filterless photometry of HS $0642+5049$. In order to achieve a high time resolution, windowing and binning $2 \times 2$ were applied. The data were reduced using MIDAS in the same manner as described for the Kryoneri run. The apparent magnitudes were extracted using the comparison star "C3", resulting in a mean magnitude of $\simeq 15.3$.

Calar Alto Observatory. During January 2003 and October 2004 , we used CAFOS with the SITe $2 \mathrm{k} \times 2 \mathrm{k}$ pixel CCD camera on the $2.2 \mathrm{~m}$ telescope to obtain filterless differential photometry of HS $0506+7725$ and HS $0642+5049$ when the atmospheric conditions were too poor for spectroscopy. Only a small part of the CCD was read out in order to improve the time resolution. The data were reduced in an analogous fashion to the one described for the Kryoneri observations above. The mean magnitudes of HS 0506+7725 and HS $0642+5049$ were found to be $\simeq 14.9$ and $\simeq 15.6$, respectively.

Light curve morphology. The light curves of HS 0139+0559 (Fig. 6) and HS 0229+8016 (Fig. 7) display very little variability on nightly time scales, with amplitudes $\lesssim 0.02$ mag and $\sim 0.05 \mathrm{mag}$, respectively. In the case of HS $0229+8016$, a low-amplitude modulation with a period of $\sim 4 \mathrm{~h}$ is consistently detected during the two longest observations. HS 0506+7725 exhibits short-period variability with an amplitude of $\sim 0.2-0.4 \mathrm{mag}$, which appear to be quasi-periodic oscillations on time scales of $\sim 10-20 \mathrm{~min}$ (Fig. 8). No clearly repeating variation is detected on time scales of several hours (i.e. a putative orbital modulation). The light curves of HS 0642+5049 from the IAC80 (Fig. 9) show modulation with a period of $\sim 3.5 \mathrm{~h}$, which we interpret as the orbital period of the system. No substantial flickering activity is detected.
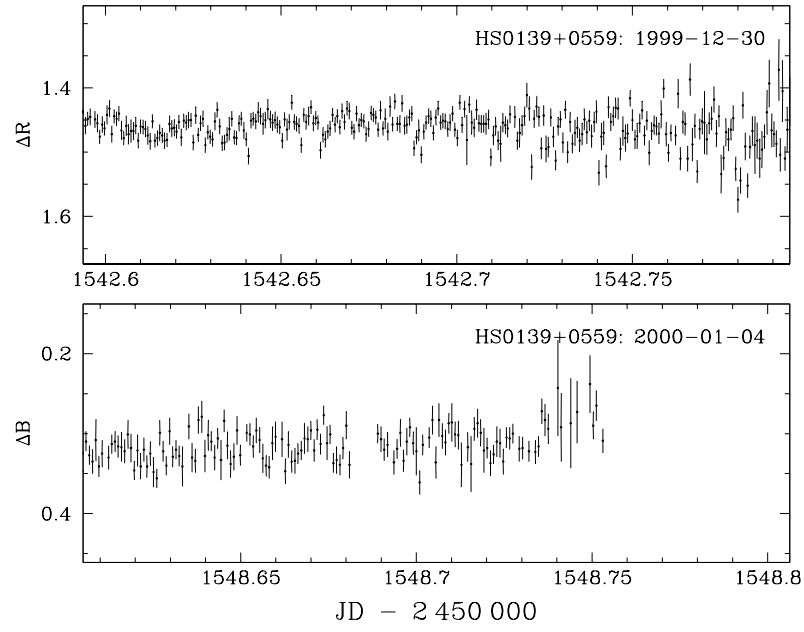

Fig. 6. Differential CCD $R$-band (top panel) and $B$-band (bottom panel) photometry of HS 0139+0559 obtained at the Braeside observatory.

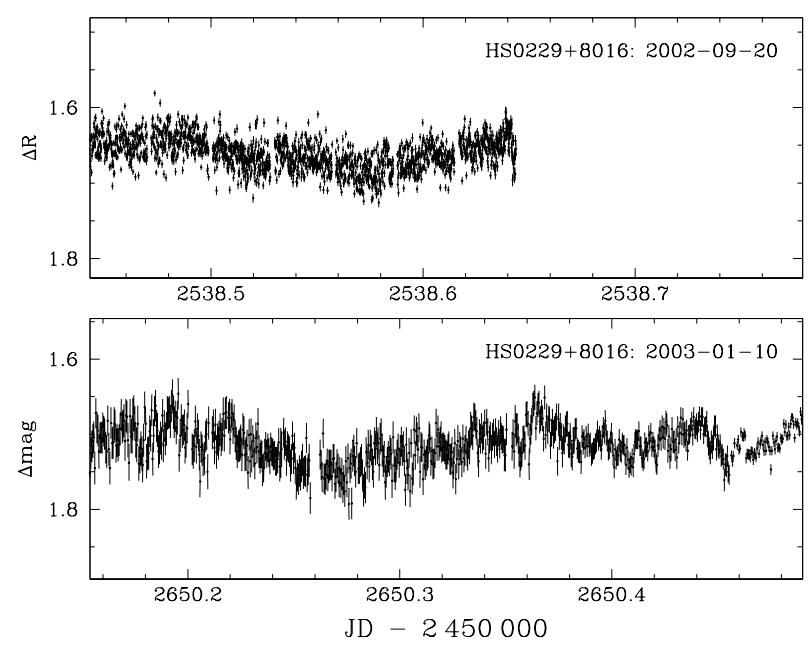

Fig. 7. Sample light curves of HS $0229+8016$. Top panel: $R$-band data obtained at the Kryoneri observatory. Bottom panel: filterless data obtained at the Tuorla observatory.

The USNO-A2.0 catalogue lists HS $0139+0559$ with $B=$ $R=14.4$, and we found $V \simeq 15.4$ and $B \simeq 14.9$ in our observations. HS $0229+8016$ has $B=13.9$ and $R=13.8$ in the USNO-A2.0 catalogue, and it was found during our observations mostly near $B \simeq V \simeq 14.0-14.6$, except on one occasion (August 1992) when it was as faint as $V \simeq 15.0$ mag. HS $0506+7725$ is listed with $B=15.3$ and $R=15.6$ in the USNO-A2.0 catalogue, and our data provides evidence of long-term variability of the mean magnitude by $\sim 1 \mathrm{mag}$, but it dropped on one occasion (October 1995) into a deep low state at $B \simeq 18.3$. HS $0642+5049$ is found in the USNOA2.0 catalogue with $B=16.6$ and $R=16.9$, and we found $V \simeq 15.3-15.5 \mathrm{mag}$ without significant long-term variability of the system. 
Table 2. Sine fits to the $\mathrm{H} \alpha$ emission line radial velocities. The methods employed were a convolution with a single Gaussian (SG) or Schneider \& Young's (1980) double-Gaussian prescription (DG).

\begin{tabular}{ccccccc}
\hline \hline Object & Method & $F W H M /$ Sep. $\left(\mathrm{km} \mathrm{s}^{-1}\right)$ & $T_{0}$ & Period (days) & $K\left(\mathrm{~km} \mathrm{~s}^{-1}\right)$ & $\gamma\left(\mathrm{km} \mathrm{s}^{-1}\right)$ \\
\hline HS 0139+0559 & SG & 600 & $2452998.985 \pm 0.013$ & $0.16923 \pm 0.00034$ & $84.4 \pm 4.8$ & $18.4 \pm 3.5$ \\
HS 0229+8016 & SG & 600 & $2452992.457 \pm 0.006$ & $0.161493 \pm 0.000034$ & $179.0 \pm 5.2$ & $35.5 \pm 3.5$ \\
HS 0506+7725 & DG & $700 / 1500$ & $2452990.679 \pm 0.022$ & $0.14770 \pm 0.00014$ & $42.6 \pm 4.4$ & $-66.7 \pm 3.0$ \\
\hline
\end{tabular}
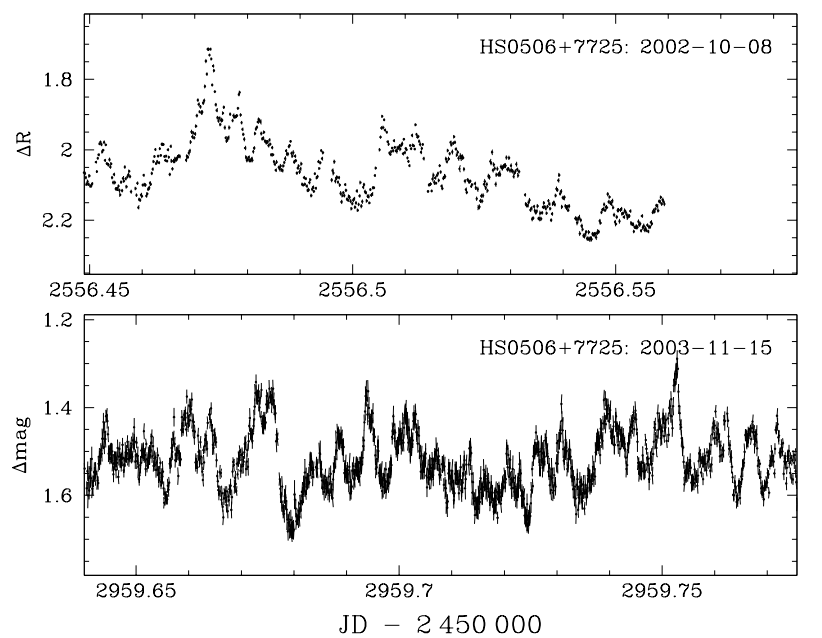

Fig. 8. Sample light curves of HS 0506+7725. Top panel: $R$-band data obtained at the Kryoneri observatory. Bottom panel: filterless data obtained at the OGS telescope.
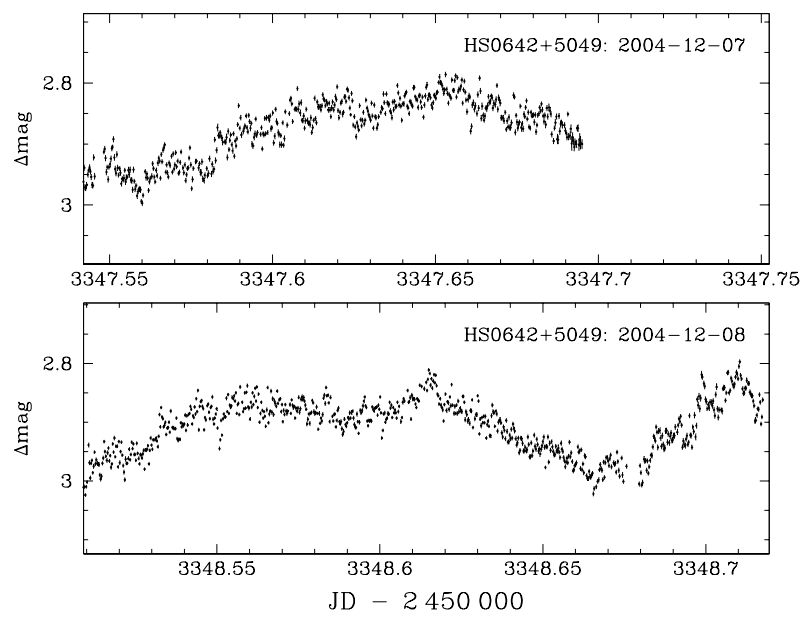

Fig. 9. Sample filterless light curves of HS $0642+5049$ obtained at the IAC80 telescope.

\section{Analysis}

\subsection{HS 0139+0559}

In order to determine the orbital period of HS 0139+0559 we measured the radial velocity variations of $\mathrm{H} \alpha$ by convolving the observed line profiles with a single Gaussion of $F W H M=$ $600 \mathrm{~km} \mathrm{~s}^{-1}$. The spectra were continuum-normalised prior to this analysis. A Scargle (1982) period analysis of the radial velocity measurements was performed using the MIDAS/TSA context. The resulting periodogram (Fig. 10) shows a strong signal at $5.909 \pm 0.012 \mathrm{~d}^{-1}$ surrounded by one-day aliases,

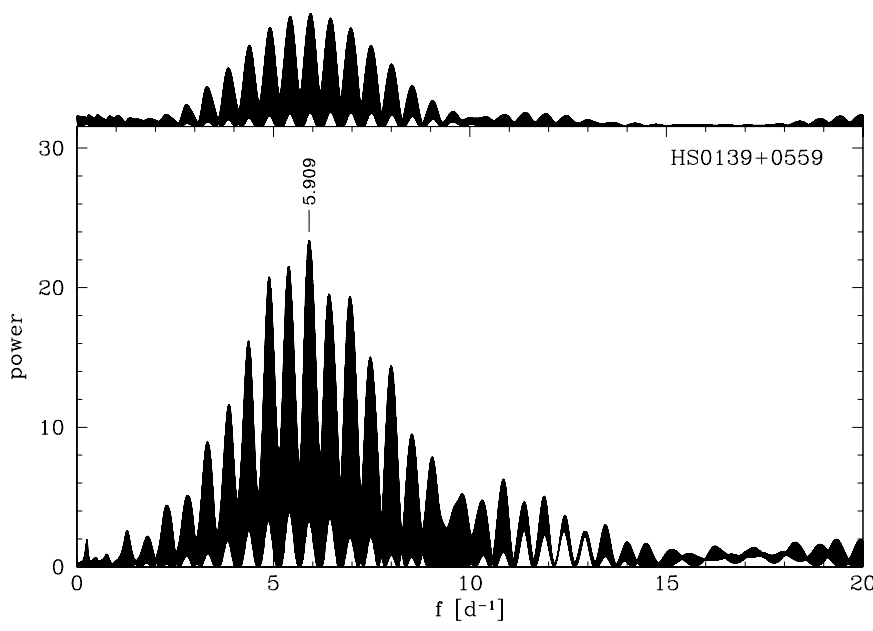

Fig. 10. The Scargle periodogram of the radial velocities of HS 0139+0559 measured from the $\mathrm{H} \alpha$ emission line. The periodogram from a set of fake radial velocities is shown in the top panel.

where the error is determined from fitting a sine wave to the radial velocity variation (see Table 2 for the full fit parameter). In order to test the significance of the detected signal, we created a set of fake radial velocities by evaluating a sine function with a frequency of $5.909 \mathrm{~d}^{-1}$ at the exact times of the observed spectroscopic data. The amplitude of the sine wave was adjusted to reflect the observed radial velocity amplitude, and the fake radial velocity measurements were randomly offset from the computed sine wave using the observed errors. The periodogram of the fake data reproduces the alias structure of the periodogram computed from the observations very well. We conclude that the orbital period of HS $0139+0559$ is $P_{\text {orb }}=243.69 \pm 0.49 \mathrm{~min}$. Folding the radial velocity measurements over that period results in a quasi-sinusoidal radial velocity curve with an amplitude of $84.4 \pm 4.8 \mathrm{~km} \mathrm{~s}^{-1}$ (Fig. 14, top panel).

For completeness, we also used the Braeside $B$ and $R$ band photometry for a period analysis. As suggested by the flat light curve (Fig. 6), no significant signal is detected in the Scargle periodogram.

\subsection{HS $0229+8016$}

An analogous radial velocity analysis as described in the previous section was carried out for HS 0229+8016. Inspection of the Scargle periodogram (Fig. 11) shows a somewhat more complex alias structure as a result of the inhomogeneous spacing of the spectroscopic observations. The strongest signal is found at $6.1922 \pm 0.0013 \mathrm{~d}^{-1}$, where the error is again 


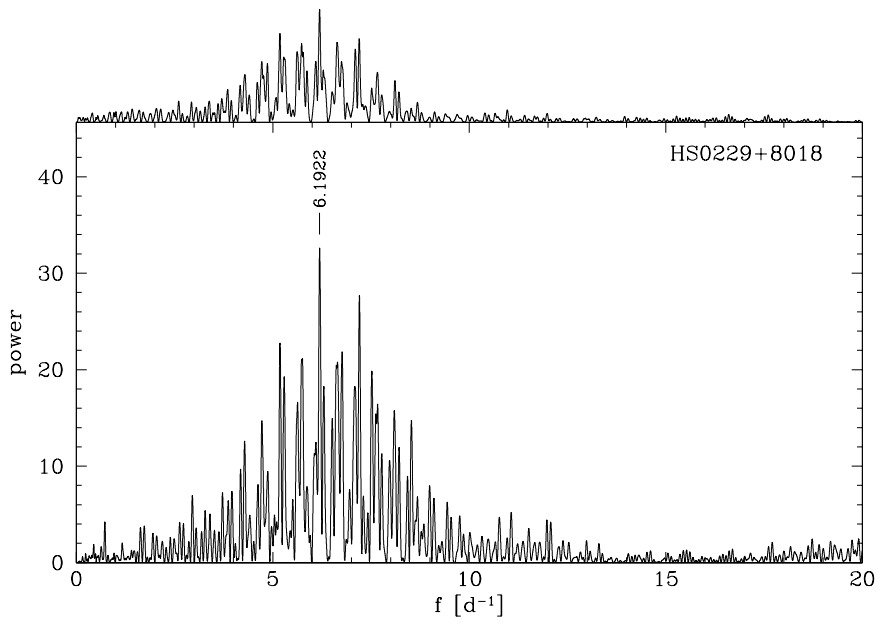

Fig. 11. The Scargle periodogram of the radial velocities of HS 0229+8016 measured from the $\mathrm{H} \alpha$ emission line. The periodogram from a set of fake radial velocities is shown in the top panel.

computed from a sine fit to the radial velocity data (Table 2), and a fake data set computed using this frequency reproduces the overall alias structure well. We conclude that the most likely value for the orbital period of HS $0229+0559$ is $P_{\text {orb }}=232.550 \pm 0.049 \mathrm{~min}$. The phase-folded radial velocity curve shows quasi-sinusoidal modulation with an amplitude of $179.0 \pm 5.2 \mathrm{~km} \mathrm{~s}^{-1}$ (Table 2).

Scargle periodograms computed from the two longest photometry runs on HS $0229+0559$ are dominated by a broad signal near $5.2 \mathrm{~d}^{-1}$ (Kryoneri data) and $6.2 \mathrm{~d}^{-1}$ (Tuorla), which are consistent with the spectroscopic period or its one-day alias. While our photometric data is not sufficient to improve the period determination of HS $0229+0559$, it suggests that the orbital period of HS $0229+0559$ can be refined by a sequence of sufficiently long photometric time-series.

\subsection{HS $0506+7725$}

Our initial analysis of the HS $0506+7725 \mathrm{H} \alpha$ radial velocity variation was carried out by applying a single-Gaussian convolution on the continuum-normalised line profiles. However, the Scargle periodogram computed from the measured radial velocity variations turned out to be dominated by a variety of signals in the range $\sim 1-5 \mathrm{~d}^{-1}$, none of which resulted in a plausible phase-folded radial velocity curve. In a second attempt, we applied the double Gaussian method of Schneider $\&$ Young (1980), using a Gaussian $F W H M=700 \mathrm{~km} \mathrm{~s}^{-1}$ and a separation of $1500 \mathrm{~km} \mathrm{~s}^{-1}$ to measure the radial velocity variation of the line wings. The Scargle periodogram resulting from these radial velocity measurements includes several peaks in the range 5-9 $\mathrm{d}^{-1}$ (Fig. 12). The strongest signal is found at $6.7706 \pm 0.0065 \mathrm{~d}^{-1}$, which we identify as the likely orbital period of HS $0506+7725, P_{\text {orb }} \simeq 212.7 \pm 0.2 \mathrm{~min}$, where the error is determined from a sine fit to the radial velocity data (Table 2). The Scargle periodogram computed from a fake data set results in a much cleaner periodogram than the one obtained from the observed data, suggesting that the line wings are affected by additional velocity contributions apart from the

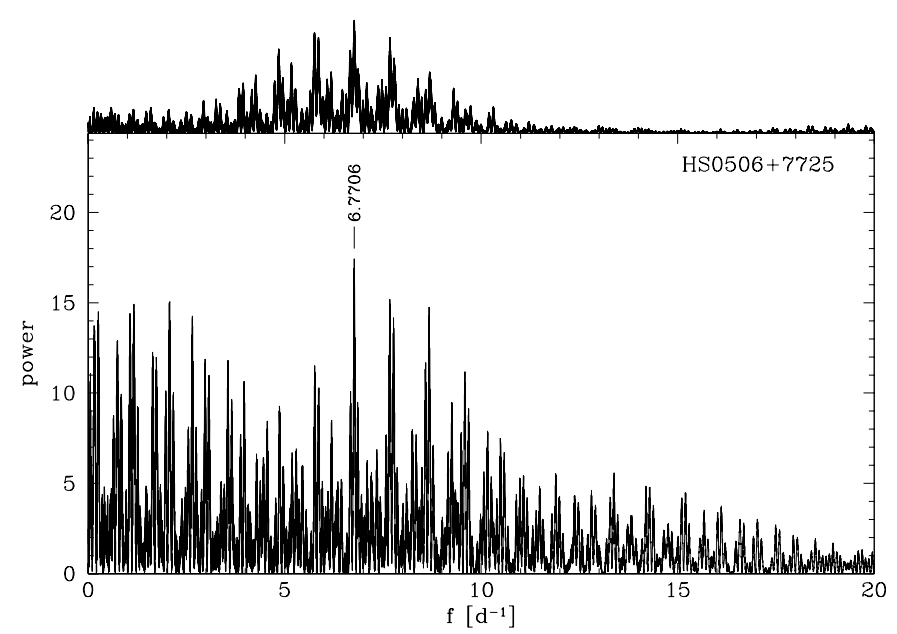

Fig. 12. The Scargle periodogram of the radial velocities of HS 0506+7725 measured from the $\mathrm{H} \alpha$ emission line by using the double Gaussian method. The periodogram from a set of fake radial velocities is shown in the top panel.

orbital motion. The $\mathrm{H} \alpha$ radial velocities folded over the orbital period (Fig. 14) display a low amplitude of $42.6 \pm 4.4 \mathrm{~km} \mathrm{~s}^{-1}$ and a relatively large amount of scatter, again suggesting that the orbital motion measured from the line wings is contaminated by another velocity component.

Our time-series analysis of the photometry of HS 0506+7725 did not lead to the detection of any significant signal, either at long (orbital) or at short (putative white dwarf spin) frequencies, making the observed shortterm variability (Fig. 8) a nice example of non-coherent $\mathrm{CV}$ flickering.

\subsection{HS $0642+5049$}

The 87 available spectra of HS $0642+5049$ were subjected to radial velocity studies as outlined above, both using the singleGaussian and double-Gaussion convolution techniques. None of the resulting Scargle periodograms contained any significant signal. Inspecting trailed spectra assembled from our data, we concluded that HS $0642+5049$ does not show any radial velocity variation at our spectral resolution.

Considering the $\sim 3.5 \mathrm{~h}$ modulation observed in the HS 0642+5049 light curves, we used the three longest and closest spaced photometric data sets obtained at the Calar Alto $2.2 \mathrm{~m}$ telescope (October 25, 2004) and the IAC80 (December 8 and 9, 2004) to determine the orbital period of the system. The strongest peak detected in the Scargle periodogram computed from these data is found at $6.3746 \pm 0.0065 \mathrm{~d}^{-1}$, surrounded by one-day aliases (Fig. 13). In order to test the significance of the signal, we created a set of fake data from a sine wave with a frequency of 6.3746 evaluated at the exact times of the observations. The alias structures of the periodograms calculated from the fake data and the real data agree well. We conclude that the orbital period of HS $0642+5049$ is $P_{\text {orb }} \simeq 225.90 \pm 0.23 \mathrm{~min}$. 


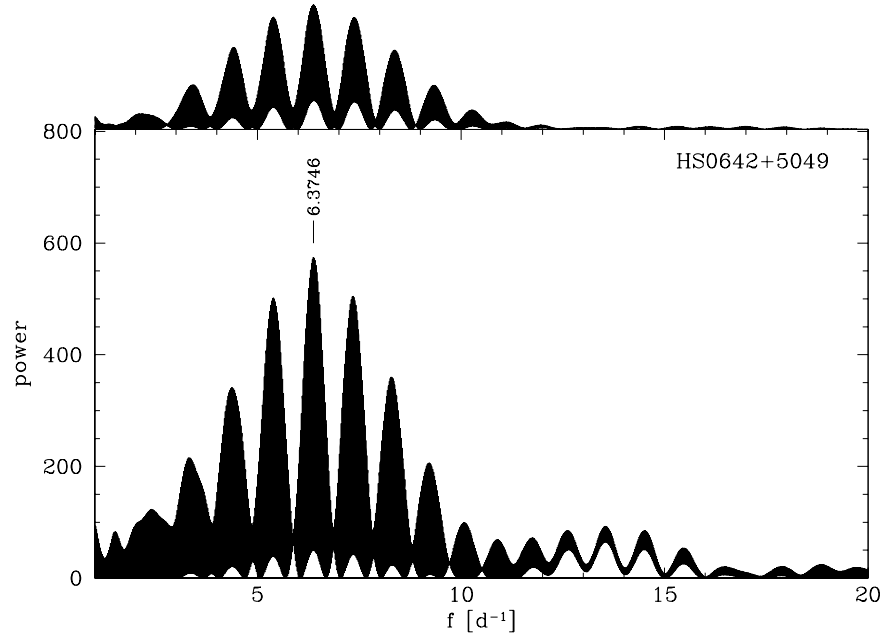

Fig. 13. The Scargle periodogram of HS $0642+5049$ computed from the three longest nights of differential photometry obtained at the Calar Alto (October 25, 2004) and the IAC80 (December 8 and 9, 2004). The periodogram from a fake data set assuming a period of $225.90 \mathrm{~min}$ is shown in the top panel.

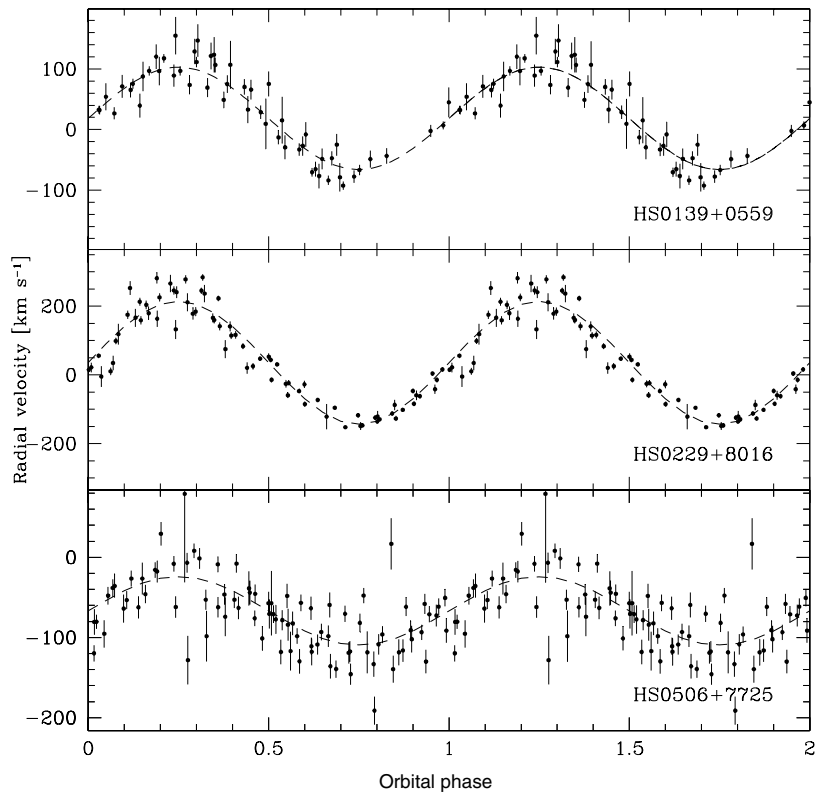

Fig. 14. $\mathrm{H} \alpha$ radial velocities of HS 0139+0559 (top panel), HS 0229+8016 (middle panel), and HS 0506+7725 (bottom panel) folded on the period of $243.69 \mathrm{~min}, 232.550 \mathrm{~min}$, and $212.7 \mathrm{~min}$, respectively. The dashed lines are the best sine fits to the folded velocities.

Figure 15 shows the CAFOS and the IAC80 photometry folded on $225.90 \mathrm{~min}$ and averaged into 30 phase bins, which reflects the morphology of the individual light curves (Fig. 9).

\section{Discussion}

\subsection{The inventory of the 3-4 h orbital period range}

The primary aim of our search for CVs in the HQS is to establish the orbital periods and $\mathrm{CV}$ subtypes for a large

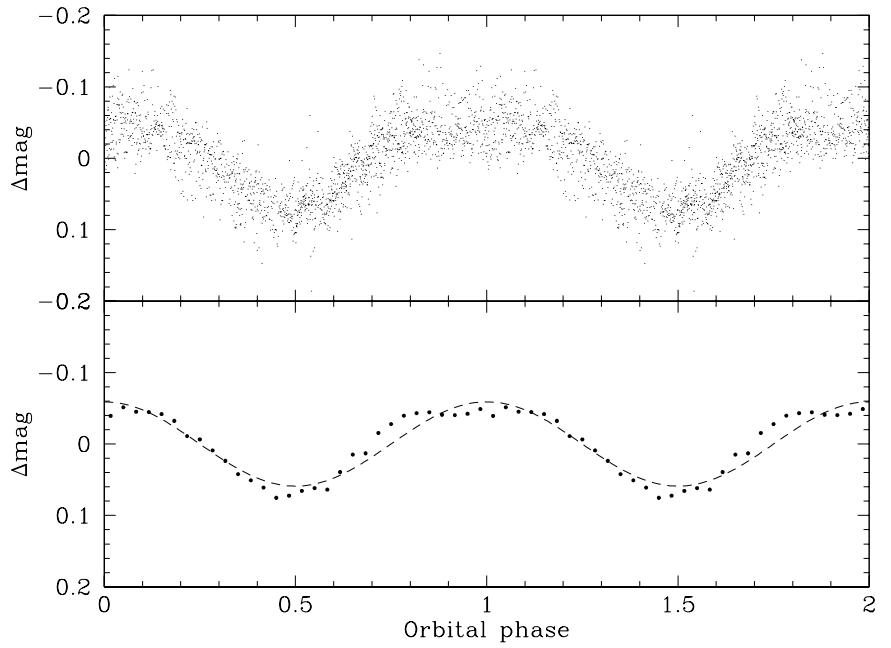

Fig. 15. HS $0642+5049$ photometric data from the CAFOS (October $9,2004)$ and the IAC80 (December 8 and 9, 2004) folded on $P_{\text {orb }}=$ $225.90 \mathrm{~min}$ (top panel). The average light curve, binned into 30 phase, is shown in the bottom panel along with a sine fit (dashed line).

sample of $\mathrm{CVs}$ that were selected in a homogeneous way based on their spectroscopic properties. The properties of this sample will then be compared with the predictions of $\mathrm{CV}$ evolution theory. Here, we report the spectroscopic identification and detailed follow-up studies of HS 0139+0559, HS 0229+8016, HS 0506+7725, and HS 0642+5049, which have the orbital periods of $243.69 \mathrm{~min}, 232.550 \mathrm{~min}, 212.7 \mathrm{~min}$, and $225.90 \mathrm{~min}$, respectively. This follows the trend noticed by Gänsicke et al. (2002b) and, more recently, by Gänsicke (2004) and Rodríguez-Gil (2005) that the majority of the new CVs identified in the HQS have orbital periods above the period gap and the bulk of them are concentrated in the 3-4 h orbital period range. Currently, orbital periods have been determined for 42 systems out of a total of 53 new HQS CVs, and Fig. 16 compares the period distribution of these new HQSCVs with the period distribution of the CVs from the Ritter \& Kolb catalogue (2003, V7.4). Even though the followup of the new HQS CVs is not yet complete, it is already now clear that our survey did not identify the large number of short-period CVs predicted by the population models (e.g. Kolb 1993; Howell et al. 1997), even though our selection method (= detection of Balmer emission lines) is best suited for the identification of low mass transfer systems that might be inconspicuous in other ways (variability, X-rays), such as the shortperiod dwarf novae HS 1449+6415 (Nogami et al. 2000) and HS 2219+1824 (Rodríguez-Gil et al. 2005a), or the ultra-short period HS 2331+3905, which might be a WZ Sge-type dwarf novae with extremely long outburst recurrence times (AraujoBetancor et al. 2005a). The (somewhat preliminary) conclusion is that, if a large number of short-period CVs does indeed exist, they must look different from the well-known examples such as WZ Sge.

The HQS CV survey has been very prolific in identifying relatively bright long-period CVs with a distinct preference for the 3-4 h period range (Fig. 16), including the four 
Table 3. Comparison of the observational characteristics of the four new CVs. The (non)detection of X-ray emission refers to the ROSAT All Sky Survey (Voges et al. 2000). The CV subtypes are abbreviated as UX = UX UMa type novalike variable, ZC = ZCam type dwarf nova, $\mathrm{VY}=\mathrm{VY}$ Scl star.

\begin{tabular}{|c|c|c|c|c|c|c|c|}
\hline \multirow[t]{2}{*}{ Object } & \multirow{2}{*}{$\begin{array}{c}P_{\text {orb }} \\
{[\mathrm{min}]}\end{array}$} & \multirow{2}{*}{$\begin{array}{c}\text { Radial velocity } \\
\text { variation }\end{array}$} & \multicolumn{3}{|c|}{ Photometric variability } & \multirow[t]{2}{*}{ X-ray } & \multirow[t]{2}{*}{ type } \\
\hline & & & Orbital & Flickering & Long-term & & \\
\hline HS 0139+0559 & 243.7 & clean, moderate amplitude & none & none & none & no & $\mathrm{UX}$ or $\mathrm{ZC}$ \\
\hline HS 0229+8016 & 232.6 & clean, large amplitude & very low amplitude & none & $\sim 1.3 \mathrm{mag}$ & no & UX or ZC \\
\hline HS 0506+7725 & 212.7 & scatter, low amplitude & not obvious & large amplitude & $\sim 3$ mag low state & yes & VY \\
\hline HS 0642+5049 & 225.9 & very low amplitude & moderate amplitude & low amplitude & none & maybe & $\mathrm{UX}$ or $\mathrm{ZC}$ \\
\hline
\end{tabular}

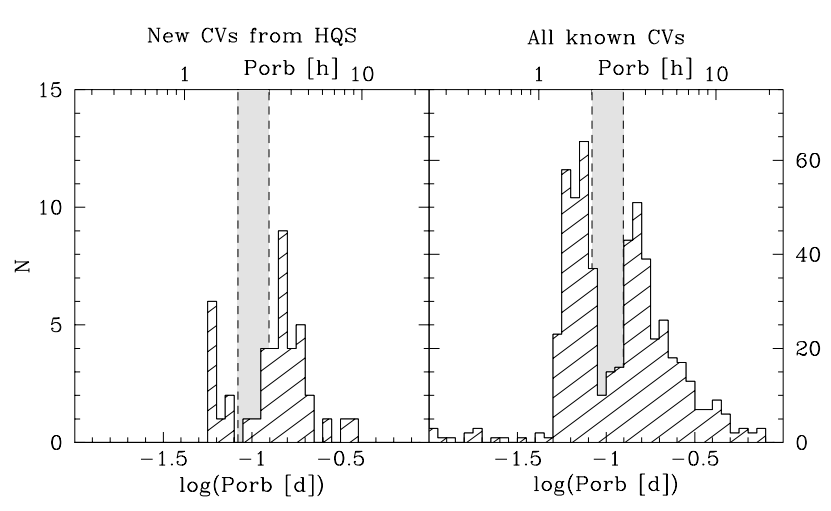

Fig. 16. The period distribution of 42 new CVs discovered in the HQS (left panel) and of all known CVs (right panel, from Ritter \& Kolb $2003, \mathrm{~V} 7.4)$. The $2-3 \mathrm{~h}$ period gap is shaded in gray.

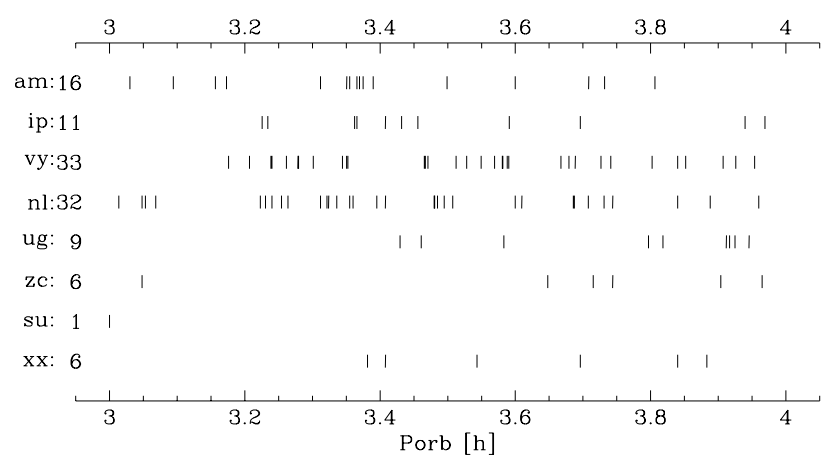

Fig. 17. The period distribution of the individual CV subtypes in the 3-4 h period range. From top to bottom: polars (am), intermediate polars (ip), VY Scl and SW Sex stars (vy), novalike variables and nova remnants that are neither VY Scl nor SW Sex stars (nl), U Gem type dwarf novae (ug), ZCam type dwarf novae (zc), SU UMa type dwarf novae (su), and systems with undetermined CV subtype (xx).

new CVs presented in this paper. The majority of these new long-period CVs are weak or no X-ray emitters, and they display little long-term variability - in fact, only five confirmed dwarf novae are among the 28 new systems found above the gap. Gänsicke (2004) and Rodríguez-Gil (2005) pointed out the large number of SW Sextantis stars among the new HQS CVs, which represent $25 \%$ of all newly identified CVs above the gap, and nearly half of all the new CVs in the 3-4 h period range. For comparison, we show in Fig. 17 the inventory of the 3-4 h orbital period range according to
Ritter \& Kolb (2003, V7.4). We find that 114 CVs (20\% of all CVs with known $P_{\text {orb }}$ ) inhabit the $3-4 \mathrm{~h}$ period range, of which 27 (24\%) are confirmed magnetic systems (intermediate polars, polars). 33 (29\%) belong to the group of either VY Scl or SW Sex stars, which share similar properties, and are suspected to contain magnetic white dwarfs as well (e.g. Rodríguez-Gil et al. 2001; Hameury \& Lasota 2002). While the ratio of definite magnetic CVs in the $3-4 \mathrm{~h}$ period range (24\%) is already very high compared to the incidence of magnetism in isolated white dwarfs (Liebert et al. 2003), confirmation of significant magnetism in the white dwarfs in VY Scl/SW Sex stars would raise the ratio of magnetic/non-magnetic $\mathrm{CVs}$ well above $50 \%$, which conflicts with any of the current models of $\mathrm{CV}$ evolution. Whatever the verdict on the magnetic fields in VY Scl/SW Sex stars will be, the large number of $\mathrm{CV}$ s belonging to this type suggests that they represent an important phase of $\mathrm{CV}$ evolution rather than some unusual combination of their physical properties. For completeness, $32(28 \%)$ novalike variables that do not belong to either the VY Scl or SW Sex class populate the 3-4 h period range ${ }^{2}$. While a number of those systems definitely do not share any of the VY Scl/SW Sex properties, a fair fraction of these systems has been studied only in a very limited way, and hence some of them might well join the VY Scl/SW Sex class upon more detailed scrutiny. Finally, 16 (14\%) dwarf novae are known in the 3-4 h period range, and the scarcity of systems undergoing thermal disc instabilities just above the period gap is a well-known fact (e.g. Shafter et al. 1986; Shafter 1992).

\subsection{The nature of the four new CVs}

Based on observational characteristics (summarised in Table 3) we discuss the likely nature of the four new CVs.

HS 0506+7725 shows short time scale flickering with quasi-periodic oscillations on time scales of $\sim 15 \mathrm{~min}$. The relatively narrow emission lines and the low amplitude of the radial velocity variations suggest a low inclination. The system has been detected in the RASS (Voges et al. 2000) at $0.07 \mathrm{cts} \mathrm{s}^{-1}$ (1RXS J051336.1+772836) with a hard spectrum, and has been previously detected as an X-ray source by EINSTEIN (2E 0506.1+7725). The presence of moderately

2 The classification of Ritter \& Kolb (2003) is somewhat confusing, as their use of the UX UMa type disagrees with the more common definition of systems charcterised by persistent broad Balmer absorption lines. 
strong He II $\lambda 4686$ emission in the identification spectrum independently confirms the presence of ionising radiation in the system. The detection of a deep low state at $B \simeq 18.3$ on one of the HQS prism plates clearly identifies the system as a VY Scl star. The system does not display evidence at face value of being an SW Sex star; but as it is obviously a low-inclination binary, a spectroscopic study at higher resolution would be useful to test for anomalous radial velocity behaviour in the emission lines.

The other three systems, HS 0139+0559, HS 0229+8016, and HS 0642+5049 are spectroscopically very similar, being characterised by thick-disc absorption line spectra. The fact that we have observed them on various occasions and found them always at nearly the same magnitude and with the same spectral properties ${ }^{3}$ makes it very unlikely that these systems are U Gem-type dwarf novae observed during outburst. While HS $0139+0559$ and HS 0229+8016 are not detected in the RASS, a faint X-ray source is found near HS 0642+5049 (1RXS J066618.4+504601, $0.02 \mathrm{cts} \mathrm{s}^{-1}$ ), which coincides within the $29^{\prime \prime}$ position error of the RASS detection with the CV. The fact that there are no other nearby objects suggests that HS $0642+5049$ is a weak X-ray emitter. None of the systems shows strong flickering activity. One puzzling difference among the three systems is that, whereas HS 0139+0559 and HS 0229+8016 show no or only very low-amplitude orbital photometric variability but exhibit clean quasi-sinusoidal radial velocity variations in their emission lines, HS 0642+5049 does not display any radial velocity variation but shows a $0.2 \mathrm{mag}$ photometric modulation. It is very difficult to reconcile this opposite difference in spectroscopic/photometric behaviour in the simple picture of a highmass transfer $\mathrm{CV}$ with a steady-state accretion disc. Based on our data, we identify all three systems either as UX UMa-type novalike variables or as Z Cam-type dwarf novae observed in periods of standstill. Optical long-term monitoring will be necessary to distinguish between these two possibilities.

\section{Conclusions}

We have identified HS 0139+0559, HS 0229+8016, HS 0506+7725, and HS 0642+5049 as long-period CVs with the orbital periods of $243.69 \pm 0.49 \mathrm{~min}, 232.550 \pm 0.049 \mathrm{~min}$, $212.7 \pm 0.2 \mathrm{~min}$, and $225.90 \pm 0.23$, respectively. HS 0506 +7725 is a VYScl novalike variable characterised by a strong emission-line spectrum. HS 0139+0559, HS 0229+8016, and HS 0642+5049 have thick-disc spectra and are either UX UMa type novalike variables or ZCam dwarf nova. None of the objects is a strong X-ray source or displays large-amplitude outbursts, which underlines the strength of CV surveys on spectroscopically selected candidates.

\footnotetext{
3 With one exception: HS 0229+8016 was observed in August 1992 in a somewhat fainter state, $V \simeq 15.0$, compared to its typical brightness near 14 mag. On that occasion, the Balmer and He I absorption lines were absent/weak, and the strength of the emission lines had markedly increased.
}

Acknowledgements. A.A. thanks the Royal Thai Government for a studentship. B.T.G. and P.R.G. were supported by a PPARC Advanced Fellowship and a PDRA grant, respectively. The HQS was supported by the Deutsche Forschungsgemeinschaft through grants Re 353/11 and $\operatorname{Re} 353 / 22$. Tom Marsh is acknowledged for developing and sharing his reduction and analysis package MOLLY. We thank the referee, Mike Shara, for his comments that lead to an improved presentation of the paper.

\section{References}

Andronov, N., Pinsonneault, M., \& Sills, A. 2003, ApJ, 582, 358

Araujo-Betancor, S., Gänsicke, B. T., Hagen, H.-J., Rodríguez-Gil, P., \& Engels, D. 2003, A\&A, 406, 213

Araujo-Betancor, S., Gänsicke, B. T., Hagen, H.-J., et al. 2005a, A\&A, 430,629

Araujo-Betancor, S., Gänsicke, B. T., Long, K. S., et al. 2005b, ApJ, 622,589

Barker, J., \& Kolb, U. 2003, MNRAS, 340, 623

Bertin, E., \& Arnouts, S. 1996, A\&AS, 117, 393

de Kool, M. 1992, A\&A, 261, 188

Downes, R. A. 1986, ApJ, 307, 170

Downes, R. A., Webbink, R. F., Shara, M. M., et al. 2001, PASP, 113, 764

Gänsicke, B. T. 2004, in Compact Binaries and Beyond, ed. G. Tovmassian \& E. Sion, Rev. Mex. Astron. Astrofis., Ser. Conf., 20, 152

Gänsicke, B. T. 2005, in The Astrophysics of Cataclysmic Variables and Related Objects, ed. J.-M. Hameury \& J.-P. Lasota, ASP Conf. Ser., 330

Gänsicke, B. T., Fried, R. E., Hagen, H.-J., et al. 2000, A\&A, 356, L79

Gänsicke, B. T., Beuermann, K., \& Reinsch, K. 2002a, The Physics of Cataclysmic Variables and Related Objects, ASP Conf. Ser., 261

Gänsicke, B. T., Hagen, H. J., \& Engels, D. 2002b, in The Physics of Cataclysmic Variables and Related Objects, ed. B. T. Gänsicke, K. Beuermann, \& K. Reinsch, ASP Conf. Ser., 261, 190

Gänsicke, B. T., Hagen, H.-J., Kube, J., et al. 2002c, in The Physics of Cataclysmic Variables and Related Objects, ed. B. T. Gänsicke, K. Beuermann, \& K. Reinsch, ASP Conf. Ser., 261, 623

Gänsicke, B. T., Araujo-Betancor, S., Hagen, H.-J., et al. 2004a, A\&A, 418, 265

Gänsicke, B. T., Jordan, S., Beuermann, K., et al. 2004b, ApJ, 613, L141

Hagen, H. J., Groote, D., Engels, D., \& Reimers, D. 1995, A\&AS, 111,195

Hameury, J. M., \& Lasota, J. P. 2002, A\&A, 394, 231

Hameury, J.-M., \& Lasota, J.-P. (eds.) 2005, The Astrophysics of Cataclysmic Variables and Related Objects, ASP Conf. Ser., 330

Heber, U., Jordan, S., \& Weidemann, V. 1991, in NATO ASIC Proc., 336, White Dwarfs, 109

Horne, K. 1986, PASP, 98, 609

Howell, S. B., Rappaport, S., \& Politano, M. 1997, MNRAS, 287, 929

King, A. R. 1988, QJRAS, 29, 1

Kolb, U. 1993, A\&A, 271, 149

Liebert, J., Bergeron, P., \& Holberg, J. B. 2003, AJ, 125, 348

Mennickent, R. E., Tovmassian, G., Zharikov, S. V., et al. 2002, A\&A, 383, 933

Nogami, D., Engels, D., Gänsicke, B. T., et al. 2000, A\&A, 364, 701

Politano, M. 1996, ApJ, 465, 338

Ringwald, F. A. 1996, in Cataclysmic Variables and Related Objects, ed. A. Evans \& J. H. Wood (Dordrecht: Kluwer), IAU Coll., 158, 89 
Ritter, H., \& Burkert, A. 1986, A\&A, 158, 161

Ritter, H., \& Kolb, U. 2003, A\&A, 404, 301

Rodríguez-Gil, P. 2005, in The Astrophysics of Cataclysmic Variables and Related Objects, ed. J.-M. Hameury \& J.-P. Lasota, ASP Conf. Ser., 330, 335

Rodríguez-Gil, P., Casares, J., Martínez-Pais, I. G., Hakala, P., \& Steeghs, D. 2001, ApJ, 548, L49

Rodríguez-Gil, P., Gänsicke, B. T., Araujo-Betancor, S., \& Casares, J. 2004a, MNRAS, 349, 367

Rodríguez-Gil, P., Gänsicke, B. T., Barwig, H., Hagen, H.-J., \& Engels, D. 2004b, A\&A, 424, 647

Rodríguez-Gil, P., Gänsicke, B. T., Hagen, H.-J., et al. 2005a, A\&A, 431, 269

Rodríguez-Gil, P., Gänsicke, B. T., Hagen, H.-J., et al. 2005b, A\&A, 440,701
Scargle, J. D. 1982, ApJ, 263, 835

Schenker, K., \& King, A. R. 2002, in The Physics of Cataclysmic Variables and Related Objects, ed. B. T. Gänsicke, K. Beuermann, \& K. Reinsch, ASP Conf. Ser., 261, 242

Schneider, D. P., \& Young, P. 1980, ApJ, 238, 946

Shafter, A. W. 1992, ApJ, 394, 268

Shafter, A. W., Wheeler, J. C., \& Cannizzo, J. K. 1986, ApJ, 305, 261

Szkody, P., Gänsicke, B. T., Fried, R. E., Heber, U., \& Erb, D. K. 2001, PASP, 113, 1215

Szkody, P., Henden, A., Fraser, O. J., et al. 2005, AJ, 129, 2386

Taam, R. E., Sandquist, E. L., \& Dubus, G. 2003, ApJ, 592, 1124

Thorstensen, J. R., \& Fenton, W. H. 2002, PASP, 114, 74

Voges, W., Aschenbach, B., Boller, T., et al. 2000, IAU Circ., 7432 
A. Aungwerojwit et al.: Four new long-period cataclysmic variables, Online Material p 1

\section{Online Material}


A. Aungwerojwit et al.: Four new long-period cataclysmic variables, Online Material $p 2$

Table 1. Log of the observations.

Table 1. continued.

\begin{tabular}{|c|c|c|c|c|c|c|c|c|c|c|c|}
\hline Date & UT time & $\begin{array}{c}\text { Filter/ } \\
\text { Grating }\end{array}$ & $\begin{array}{l}\text { Exp. } \\
\text { (s) }\end{array}$ & Frames & $\begin{array}{c}\text { Mean } \\
\text { mag }\end{array}$ & Date & UT time & $\begin{array}{l}\text { Filter/ } \\
\text { Grating }\end{array}$ & $\begin{array}{l}\text { Exp. } \\
\text { (s) }\end{array}$ & Frames & $\begin{array}{c}\text { Mean } \\
\text { mag }\end{array}$ \\
\hline \multicolumn{6}{|c|}{ HS 0139+0559 } & \multicolumn{6}{|c|}{ Spectroscopy: Hamburg-Schmidt telescope at Calar Alto } \\
\hline \multicolumn{6}{|c|}{ Spectroscopy: Calar Alto $3.5 \mathrm{~m}$ and $\mathrm{B}$ and $\mathrm{C}$} & 1987 Oct. 29 & - & Prism & 3600 & 1 & $\simeq 15.1$ \\
\hline 1989 Jan. 22 & $19: 21$ & $120 \AA / \mathrm{mm}$ & 900 & 1 & - & 1994 Jan. 12 & - & Prism & 3600 & 1 & $\simeq 15.3$ \\
\hline \multicolumn{6}{|c|}{ Spectroscopy: Calar Alto $3.5 \mathrm{~m}$ and TWIN } & 1995 Oct. 23 & - & Prism & 3600 & 1 & $\simeq 18.3$ \\
\hline 2002 Oct. 28 & $23: 53-00: 03$ & T08/T01 & 300 & 1 & - & \multicolumn{6}{|c|}{ Photometry: Kryoneri Observatory } \\
\hline 2002 Oct. 29 & $22: 36-03: 21$ & T05/T06 & 600 & 16 & - & 2002 Oct. 08 & $22: 46-01: 25$ & $R$ & 10 & 599 & $\simeq 15.2$ \\
\hline \multicolumn{6}{|c|}{ Spectroscopy: Calar Alto $2.2 \mathrm{~m}$ and CAFOS } & \multicolumn{6}{|c|}{ Photometry: Tuorla Observatory } \\
\hline 2003 Dec. 24 & $18: 25-00: 10$ & G-100 & 600 & 27 & $\simeq 15.4$ & 2003 Jan. 04 & $16: 13-20: 16$ & Clear & 120 & 32 & $\simeq 15.7$ \\
\hline 2003 Dec. 26 & $18: 34-20: 48$ & G-100 & 600 & 12 & $\simeq 15.1$ & 2003 Jan. 06 & $15: 47-20: 46$ & Clear & 60 & 213 & $\simeq 15.8$ \\
\hline \multicolumn{6}{|c|}{ Spectroscopy: Hamburg-Schmidt telescope at Calar Alto } & 2003 Jan. 16 & $20: 18-22: 40$ & Clear & 60 & 115 & $\simeq 15.9$ \\
\hline 1981 Nov. 01 & - & Prism & 3600 & 1 & $\simeq 14.9$ & \multicolumn{6}{|c|}{ Photometry: OGS } \\
\hline 1981 Nov. 02 & - & Prism & 3600 & 1 & $\simeq 14.9$ & 2003 Nov. 15 & $03: 21-06: 37$ & Clear & 5 & 1110 & $\simeq 14.6$ \\
\hline \multicolumn{6}{|c|}{ Photometry: Braeside Observatory } & \multicolumn{6}{|c|}{ Photometry: Calar Alto $2.2 \mathrm{~m}$ and CAFOS } \\
\hline 1999 Dec. 30 & 02:15-07:04 & $R$ & 70 & 293 & - & 2003 Jan. 01 & 01:01-03:24 & V & 30 & 85 & $\simeq 15.1$ \\
\hline 2000 Jan. 04 & 02:31-06:04 & $B$ & 50 & 136 & - & 2003 Dec. 16 & 00:05-05:11 & Clear & $15-30$ & 346 & $\simeq 14.8$ \\
\hline \multicolumn{6}{|c|}{ HS 0229+8016 } & 2003 Dec. 25 & $19: 15-02: 29$ & Clear & $15-30$ & 296 & $\simeq 14.9$ \\
\hline \multicolumn{6}{|c|}{ Spectroscopy: Calar Alto $2.2 \mathrm{~m}$ and $\mathrm{B}$ and C } & 2003 Dec. 27 & 04:03-06:16 & Clear & 20 & 149 & $\simeq 14.9$ \\
\hline 1992 Aug. 08 & $03: 16$ & & 900 & 1 & $\simeq 15.0$ & 2003 Dec. 27 & $20: 33-23: 55$ & Clear & 20 & 288 & $\simeq 14.8$ \\
\hline \multicolumn{6}{|c|}{ Spectroscopy: Calar Alto $2.2 \mathrm{~m}$ and CAFOS } & \multicolumn{6}{|c|}{ HS $0642+5049$} \\
\hline 1998 Oct. 05 & $01: 37$ & B-200 & 1800 & 1 & $\simeq 13.7$ & \multicolumn{6}{|c|}{ Spectroscopy: Calar Alto $2.2 \mathrm{~m}$ and CAFOS } \\
\hline 2003 Dec. 14 & $19: 18-00: 55$ & G-100 & 600 & 24 & $\simeq 14.2$ & 1999 Mar. 07 & 19:11 & B-400 & 300 & 1 & - \\
\hline 2003 Dec. 16 & 20:21-03:08 & G-100 & 600 & 27 & - & 2003 Apr. 27 & $20: 03-21: 53$ & G-100 & 600 & 9 & $\simeq 15.6$ \\
\hline 2003 Dec. 23 & 18:49-21:01 & G-100 & 600 & 12 & $\simeq 14.5$ & 2003 May 10 & $20: 20-21: 21$ & G-100 & 600 & 6 & - \\
\hline 2003 Dec. 26 & $21: 34-22: 53$ & G-100 & 600 & 7 & $\simeq 14.1$ & 2003 May 11 & $20: 17-21: 20$ & G-100 & 600 & 6 & - \\
\hline 2003 Dec. 27 & 18:46-19:22 & G-100 & 600 & 4 & $\simeq 13.7$ & 2003 Dec. 25 & $00: 56-05: 35$ & G-100 & 600 & 24 & $\simeq 15.5$ \\
\hline \multicolumn{6}{|c|}{ Spectroscopy: Hamburg-Schmidt telescope at Calar Alto } & 2003 Dec. 27 & 02:50-03:01 & G-100 & 600 & 2 & $\simeq 15.3$ \\
\hline 1986 Nov. 05 & - & Prism & 3600 & 1 & $\simeq 14.4$ & 2004 Oct. 24 & $02: 12-05: 26$ & G-100 & 600 & 17 & $\simeq 15.3$ \\
\hline 1986 Nov. 06 & - & Prism & 3600 & 1 & $\simeq 14.2$ & 2004 Oct. 26 & $04: 25-05: 31$ & G-100 & 600 & 7 & $\simeq 15.4$ \\
\hline 1994 Nov. 12 & - & Prism & 3600 & 1 & $\simeq 14.6$ & \multicolumn{6}{|c|}{ Spectroscopy: INT $2.5 \mathrm{~m}$ and IDS } \\
\hline \multicolumn{6}{|c|}{ Photometry: Kryoneri Observatory } & 2003 Apr. 25 & $21: 21-22: 14$ & $\mathrm{R} 632 \mathrm{~V}$ & 600 & 6 & - \\
\hline 2002 Sep. 20 & $01: 30-03: 28$ & $R$ & 5 & 840 & $\simeq 14.2$ & 2003 Apr. 26 & $21: 17-21: 49$ & $\mathrm{R} 632 \mathrm{~V}$ & $600-900$ & 4 & - \\
\hline 2002 Sep. 20 & $22: 37-03: 27$ & $R$ & 5 & 1680 & $\simeq 14.3$ & 2003 Apr. 28 & $21: 15-22: 08$ & $\mathrm{R} 632 \mathrm{~V}$ & 600 & 6 & - \\
\hline \multicolumn{6}{|c|}{ Photometry: Tuorla Observatory } & Spectroscopy & Hamburg-Sc & Imidt tel & scope at $\mathrm{C}$ & lar Alto & \\
\hline 2003 Jan. 10 & $15: 41-23: 45$ & Clear & 30 & 695 & $\simeq 14.3$ & 1991 Nov. 10 & - & Prism & 3600 & 1 & $\simeq 15.8$ \\
\hline & HS & $506+7725$ & & & & 1993 Oct. 24 & - & Prism & 3600 & 1 & $\simeq 16.0$ \\
\hline Spectroscopy & Calar Alto 3. & $\mathrm{~m}$ and $\mathrm{TW}$ & & & & Photometry: & Tuorla Observ & tory & & & \\
\hline 2002 Dec. 03 & 03:22-05:09 & T05/T06 & 600 & 10 & - & 2003 Dec. 30 & 19:58-21:06 & Clear & 120 & 46 & $\simeq 15.4$ \\
\hline Spectroscopy & Calar Alto 2. & $\mathrm{~m}$ and $\mathrm{CA}$ & OS & & & 2004 Jan. 01 & $18: 37-23: 27$ & Clear & 60 & 250 & $\simeq 15.5$ \\
\hline 1998 Feb. 02 & $19: 43$ & B-400 & 180 & 1 & - & Photometry: & Calar Alto 2.2 & $\mathrm{~m}$ and $\mathrm{C}$ & FOS & & \\
\hline 2002 Dec. 09 & 00:19-00:30 & G-100 & 600 & 2 & $\simeq 15.4$ & 2003 Dec. 26 & $04: 20-06: 14$ & Clear & 20-30 & 149 & $\simeq 15.6$ \\
\hline 2003 Dec. 13 & $23: 25-03: 53$ & G-100 & 600 & 23 & $\simeq 14.7$ & 2004 Oct. 22 & $04: 01-05: 25$ & Clear & 15 & 59 & $\simeq 15.7$ \\
\hline 2003 Dec. 15 & $01: 34-06: 13$ & G-100 & 600 & 23 & $\simeq 14.9$ & 2004 Oct. 25 & $01: 42-05: 51$ & Clear & $10-15$ & 463 & $\simeq 15.5$ \\
\hline 2003 Dec. 17 & $03: 40-05: 57$ & G-100 & 600 & 12 & - & Photometry: & $\mathrm{AC80}$ & & & & \\
\hline 2003 Dec. 23 & $22: 19-00: 31$ & G-100 & 600 & 12 & $\simeq 14.9$ & 2004 Dec. 02 & 04:01-05:00 & Clear & 10 & 150 & $\simeq 15.4$ \\
\hline 2003 Dec. 26 & $02: 52-03: 28$ & G-100 & 600 & 4 & $\simeq 14.9$ & 2004 Dec. 07 & 05:10-06:11 & Clear & 15 & 139 & $\simeq 15.3$ \\
\hline & & & & & & $\begin{array}{l}2004 \text { Dec. } 08 \\
2004 \text { Dec. } 09\end{array}$ & $\begin{array}{l}01: 00-04: 41 \\
00: 13-05: 14\end{array}$ & $\begin{array}{l}\text { Clear } \\
\text { Clear }\end{array}$ & $\begin{array}{l}10 \\
10\end{array}$ & $\begin{array}{l}528 \\
738\end{array}$ & $\begin{array}{l}\simeq 15.3 \\
\simeq 15.3\end{array}$ \\
\hline
\end{tabular}

